\title{
Beobachtung der totalen Mondfinsterniss am 27. Dec. 1898
}

\section{auf der Sternwarte in Bamberg von Dr. Ernst Hartwig.}

Die Sternbedeckungen während der Mondfinsterniss am 27. December 1898 konnten hier nach dem Pulkowaer Programm bei durchsichtiger, aber sehr unruhiger Luft trotz der Helligkeit der Mondscheibe ohne Schwierigkeiten am Refractor von $265 \mathrm{~mm}$ Oeffnung mit 100-facher Vergrösserung, dessen Meereshöhe $299 \mathrm{~m}$ beträgt, gut beobachtet werden, während am Heliometer die Bildbeschaffenheit keine Messungen zu. liess. Mit einer am Refractor montirten Steinheil'schen 4zölligen Portraitlinse wurde 12 Minuten vor und I I Minuten nach der Totalität je eine photographische Aufnahme gemacht, die beide die Schattengrenze nicht bestimmter auffassen lassen, als Ocularbeobachtungen.

An Eintritten (I) und Austritten (E) wurden erhalten:

BD. Nr. Ph. M. Z. Bamb.

$+22.1368 \quad 28$ I I I $^{\mathrm{h}} 34^{\mathrm{m}} 9^{\mathrm{s}} .07$ gut

+23.1392
+24

+22.136929 I 439.05 gut

$\begin{array}{r}-23.1398 \quad 39 \quad I \quad 4518.55 \\ \hline\end{array}$

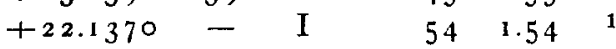

$+23.140245 \quad I \quad 553.49$

$+22.1370$

I $23 \quad 35$ schon da

$\begin{array}{lll}5 & 1.52 & 2\end{array}$

BD.

$+23.1392$

$+23.1389$

$+22.1385$

$+23.1391$

$+23.1407$

$-$

$+23.1398$

$+22.1392$

$+23.1402$

$+23.1407$

941.91

1550.30 sehr gut

$+22.136929 \mathrm{E}$

$+22.136828 \mathrm{E}$

1) schon ausgetreten

2) vielleicht um $\mathbf{I}^{\mathrm{s}}$ verzählt

Gesichtsfeldes.

Die Totalität war um $13^{\mathrm{h}} \mathrm{I}^{\mathrm{m}}$. $\mathrm{r}$ zu Ende. Vollständige Zeitbestimmungen wurden am 27. und 3r. Dec. erhalten.

Beobachtungen des Planeten $1899 \mathrm{EE}$.

\begin{tabular}{|c|c|c|c|c|c|c|c|c|c|c|}
\hline 1899 & Mittl. Ortsz. & $\Delta a$ & $\Delta \delta$ & Vgl. & $a$ app. & $\log p \cdot A$ & $\delta$ app. & $|\log p \cdot A|$ & Red. ad l. app. & $*$ \\
\hline
\end{tabular}

Auf der Sternwarte des Collegio Romano in Rom von Prof. E. Millosevich.

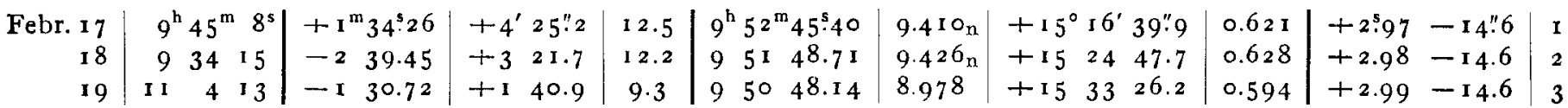

Micrometro filare. Ingrandimento 200.

Auf der k. k. Sternwarte in Wien von Dr. $\mathscr{F}$. Palisa.

Febr. $18 \mid$ I 2 I $849|+3 \quad 3.87| \rightarrow 033.9|-| 95142.51|8.591|+152538.1|0.678|+2.98-14.4 \mid 4$

Mittlere Oerter der Vergleichsterne.

\begin{tabular}{|c|c|c|c|c|c|c|c|}
\hline$*$ & $\alpha \mathbf{I} 899.0$ & $\delta 1899.0$ & Autorität & $*$ & $\alpha \mathbf{1 8 9 9 . 0}$ & $\delta 1899.0$ & Autorität \\
\hline $\mathbf{I}$ & $9^{\mathrm{h}} 5^{\mathrm{I}^{\mathrm{m}}} \quad 8^{\mathrm{s}} \mathrm{I} 7$ & + I $5^{\circ}$ I $2^{\prime} 29^{\prime \prime} 3$ & AG. Berl. A. $3985^{*}$ ) & 3 & $9^{\mathrm{h}} 5^{2^{\mathrm{m}}}$ I $5^{5} \cdot 87$ & + I $5^{\circ} 32^{\prime} \quad 0^{\prime \prime} 9$ & AG. Berl. A. $399^{2}$ \\
\hline 2 & 95425.18 & +152140.6 & AG. Berl. A. $400 \mathrm{I}$ & 4 & $9 \quad 48 \quad 35.66$ & + I 52626.4 & Neue Wien. Zonen \\
\hline
\end{tabular}

*) senza m. p.

Photographische Beobachtungen der Planeten 1899 EE und EF.

Planet I $899 \mathrm{EE}$.

\begin{tabular}{|c|c|c|c|}
\hline 1899 & M. Z. K. & $\alpha$ I 899.0 & $\delta \times 899.0$ \\
\hline $\begin{array}{r}\text { Febr. } 15 \\
\text { I } 6\end{array}$ & $\begin{array}{l}10^{\mathrm{h}} \quad 4^{\mathrm{m}} \cdot 7 \\
11 \quad 16.6\end{array}$ & $\begin{array}{l}9^{\mathrm{h}} 54^{\mathrm{m}} 35^{\mathrm{s}} \cdot 65 \\
95434.83\end{array}$ & $\begin{array}{l}+15^{\circ} 0^{\prime} 15^{\prime \prime} .0 \\
+15996.7\end{array}$ \\
\hline
\end{tabular}

Planet $1899 \mathrm{EF}$.

\begin{tabular}{|c|c|c|c|}
\hline 1899 & M. Z.K. & $a 1899.0$ & $\delta \times 899.0$ \\
\hline ebr. I 7 & $12^{\mathrm{h}} 20^{\mathrm{m}} \cdot 7$ & $10^{\mathrm{h}} 17^{\mathrm{m}} 5^{8.8}{ }^{\mathrm{s}}$ & $+7^{\circ} 13^{\prime} 9^{\prime \prime} 8$ \\
\hline 18 & I 252.2 & $\begin{array}{lll}\text { 10 } & 17 & 0.56\end{array}$ & $+721 \quad 7.6$ \\
\hline 19 & II 45.2 & I $16 \quad 7.22$ & +72846.2 \\
\hline
\end{tabular}

Die dritte Position des Planeten 1899 EF war nur unsicher zu bestimmen, da die Platte durch den Mondschein bereits stark geschwärzt war und sich das Object nur schwer im Mikroskop einstellen liess.

Königstuhl, Heidelberg, I 899 Febr. 23.

Dr. A. Schwassmann. 